\title{
Subjective Sleep Disturbance in People with Epilepsy: Prevalence and Impact on Health-Related Quality of Life
}

\author{
Sang-Ahm Lee, $\mathrm{MD}^{1}$, Young-Joo No, $\mathrm{MD}^{2}$, Kwang-Deog Jo, $\mathrm{MD}^{2}$, Jee-Hyun Kwon, $\mathrm{MD}^{3}$, \\ Jeong Yeon Kim, MD', Su-Hyun Han, MD${ }^{1}$, Dong-Jin Shin, $\mathrm{MD}^{5}$ \\ 'Department of Neurology, Asan Medical Center, University of Ulsan College of Medicine, Seoul, Korea \\ 2Department of Neurology, Gangneung Asan Hospital, University of Ulsan College of Medicine, Gangneung, Korea \\ ${ }^{3}$ Department of Neurology, Ulsan University Hospital, University of Ulsan College of Medicine, Ulsan, Korea \\ ${ }^{4}$ Department of Neurology, Sanggye Paik Hospital, Inje University College of Medicine, Seoul, Korea \\ ${ }^{5}$ Department of Neurology, Gil Medical Center, Gachon Medical School, Incheon, Korea
}

\begin{abstract}
Background and Objective It is not well known whether sleep disturbances affect quality of life (QoL) independent of mood disturbance in people with epilepsy. The aim of this study was to investigate the prevalence of sleep disturbances and the impact on QoL in people with epilepsy.

Methods This was a cross-sectional study involving adults with epilepsy and controls. Sleep disturbances, depression, anxiety, and QoL were assessed using several questionnaires. The direct effect of sleep disturbance on QoL was assessed using multiple linear regression analysis, and a mediational model designed with the assumption that sleep disturbances affect QoL through a mediator was tested.

Results A total of 168 people with epilepsy and 56 controls were enrolled. Difficulty maintaining sleep (16.1\%) and waking up too early in the morning $(13.1 \%)$ were more common in patients than controls $(\mathrm{p}<0.05)$. There were no differences in daytime sleepiness, sleep apnea, and restless legs syndrome between the groups. Patients had more sleep problems in the Medical Outcomes Study-Sleep Scale than controls. The effect of sleep disturbance on Quality of Life in Epilepsy 10 (QOLIE-10) lost its statistical significance $(\beta=-0.021, \mathrm{p}=$ 0.769) after controlling for Hospital Anxiety and Depression Scale (HADS). The Sobel test confirmed that the effect of sleep disturbance on QOLIE-10 was significantly mediated by both HADS-depression $(\beta=-0.195, \mathrm{p}<0.001)$ and HADS-anxiety $(\beta=-0.265, \mathrm{p}<0.001)$.

Conclusions Sleep disturbances, especially insomnia, were more common in people with epilepsy. Although sleep disturbance seems to have no direct effects on QoL, it appears to have an indirect effect on QoL through depression and anxiety in people with epilepsy.
\end{abstract}

Sleep Med Res 2015;6(1):16-23

Key Words Epilepsy, Sleep disturbance, Insomnia, Quality of life, Depression.

\author{
Received: November 8, 2014 \\ Revised: December 10, 2014 \\ Accepted: December 22, 2014 \\ Correspondence \\ Sang-Ahm Lee, MD \\ Department of Neurology, \\ Asan Medical Center, \\ University of Ulsan College of Medicine, \\ 88 Olympic-ro 43-gil, Songpa-gu, \\ Seoul 138-736, Korea \\ Tel +82-2-3010-3445 \\ Fax +82-2-474-4691 \\ E-mail_salee@amc.seoul.kr
}

\section{INTRODUCTION}

Sleep is essential to the health and quality of life (QoL) of all individuals and is of particular relevance to people with epilepsy. The reciprocal interaction between sleep and epilepsy was recognized long ago. Sleep disturbances such as poor sleep quality, sleep deprivation and/or obstructive sleep apnea (OSA) could easily provoke seizure recurrence, whereas epilepsy may disrupt sleep, either directly through seizures and epileptiform activity, or indirectly through medication-related effects. ${ }^{1}$

Some case-control studies found that poor sleep quality, difficulty sleeping, and/or excessive daytime sleepiness (EDS) are two to three times more common in people with epilepsy than in healthy controls. ${ }^{2-4}$ Khatami et al., ${ }^{5}$ however, found that sleep-wake habits and the frequency of most sleep disorders are similar in non-selected adults with epilepsy compared to healthy controls. The results from these studies are often not directly comparable, as the studies often used different questionnaires and heterogeneous patient populations, and were often complicated by the presence of various antiepileptic drugs (AEDs). ${ }^{1}$ 
Although the prevalence of sleep disturbances in people with epilepsy has been reported to vary depending on study population and methods to detect their sleep problems, sleep disturbance was consistently found to be an important predictor of QoL in people with epilepsy. ${ }^{3,6,7}$ However, it is unclear whether sleep disturbances affect QoL independent of mood disturbance. The strong relationship between depression and QoL has been well demonstrated in previous studies. ${ }^{8,9}$ For example, depression has a greater negative impact on QoL than other clinical indicators such as seizure frequency. ${ }^{8,10}$ In light of the strong association between mood and sleep disturbances, they may be interdependent in their influence on QoL in people with epilepsy. ${ }^{6}$ We addressed this possibility by assuming that the effects of sleep disturbance on QoL are mediated by depression or anxiety, with a greater influence on QoL and analyzed our data using linear regression and a mediation test.

The purposes of this study were 1) to explore sleep disturbances in people with epilepsy and to assess whether sleep disturbances in this population are more frequent than in the control group, 2) to determine which clinical seizure variables are associated with sleep disturbances, and 3) to assess the direct or indirect effects of sleep disturbances on QoL in people with epilepsy.

\section{METHODS}

People with epilepsy were recruited from five university hospitals in Korea over a period of 2 months. All participants were selected at the outpatient clinics for inclusion in the study only if they were aged 18 or older, had been diagnosed with epilepsy, and had been taking one or more AEDs for at least 1 year at the time of recruitment. Epilepsy was defined as a history of two or more unprovoked seizures or a single seizure with evidence of epileptiform activity recorded by electroencephalogram or structural lesions documented by brain imaging. Subjects were excluded if they had reported a seizure in the $48 \mathrm{~h}$ before completing the questionnaires, if they were shift workers or worked later than 8 p.m., if they had a neurological deficit that affected daily living activities, if they had received treatment for active psychiatric or medical disorders, or if they were unable to read or understand the questionnaires.

People with epilepsy who fulfilled the criteria and agreed to participate in this study were asked to complete a set of sleep questionnaires on the day they visited their neurologists at the outpatient clinic. Demographic and clinical data were collected by interviews and from information in medical files, and were used as confounding variables in the statistical analysis. All participants indicated whether they were regularly taking hypnotics or sedative drugs for treatment of their sleep disturbance. Demographic data were age, sex, and body mass index (BMI). Epilepsy-related data were age at seizure onset, epilepsy dura- tion, type of epilepsy (idiopathic generalized, symptomatic, cryptogenic, or undetermined epilepsy), dominant seizure type (simple partial, complex partial, or generalized tonic-clonic seizures), frequency of both generalized and focal seizures (no seizure for at least one year, 1-11/month, or monthly seizures), recurrence of generalized tonic-clonic seizure in two years, timing of seizure recurrences (nocturnal seizure defined as having more than $90 \%$ of seizures during sleep) and AED treatments (monotherapy vs. polytherapy). Written informed consent was obtained from all subjects.

Age and gender matched controls were also recruited among employees at a single university hospital, and were asked to complete a set of sleep questionnaires. The inclusion criteria were normal healthy people aged $>18$ years who had a BMI $<30 \mathrm{~kg} /$ $\mathrm{m}^{2}$, no history of medical or surgical diseases, and not currently taking any medications. Subjects who were shift workers or worked later than 8 p.m., those who slept less than 7 hours a night, or those with symptoms suspicious of cataplexy, or parasomnia were excluded.

\section{Questionnaires}

\section{Insomnia questionnaires}

Questions about insomnia were asked and respondents were required to give a yes or no answer to each of the questions. Insomnia symptoms were defined as present if a patient had any one of three insomnia complaints that lasted longer than one month and occurred at least once a week: 1) difficulty falling asleep, 2) difficulty maintaining sleep (i.e., waking up during the night and difficulty getting back to sleep), and 3) waking up too early in the morning and unable to return to sleep. ${ }^{11}$

\section{Epworth Sleepiness Scale}

The Epworth Sleepiness Scale (ESS) is the most widely used questionnaire to assess subjective daytime sleepiness. The ESS contains eight questions about how often a person dozed during day-to-day activities, answered on a 4-point scale ranging from $0=$ never dozed to $3=$ high chance of dozing. An ESS score $\geq 11$ is considered indicative of EDS. We used the Korean version of the ESS. ${ }^{12}$

\section{Sleep Apnea of Sleep Disorder Questionnaire}

The Sleep Apnea of Sleep Disorder Questionnaire (SA-SDQ) consists of eight questions and four items related to weight, smoking status, age, and BMI, which are calculated to generate a raw score. ${ }^{13}$ The eight questions address the specific symptoms of sleep apnea syndrome including snoring loudly, breathing arrest during night sleep, awaking suddenly gasping for breath, sweating during night sleep, high blood pressure, blocked nose when wanting to sleep, snoring, breathing worsening when sleeping on the back, and snoring/breathing worsening when sleeping after alcohol use. Total scores range from 0 to 60 . The sug- 
gested cut-off points for identifying Korean patients with suspected OSA are 36 for men and 30 for women. ${ }^{14}$ We used cut-off points of 29 for men and 26 for women, as has been suggested for people with epilepsy. ${ }^{15}$

\section{Restless legs syndrome questionnaire}

The questionnaire included four diagnostic criteria proposed by the International Restless Legs Syndrome (RLS) Study Group: 1) desire to move the extremities, often associated with paresthesias/dysesthesias, 2) motor restlessness, 3) worsening of symptoms at rest with at least temporary relief by activity, and 4) worsening of symptoms in the evening or at night. ${ }^{16}$ Only patients who fulfilled the four criteria for RLS were considered as having RLS.

\section{Medical Outcomes Study-Sleep Scale}

The Medical Outcomes Study (MOS)-Sleep is one of the most widely used scales for evaluating broad-spectrum sleep quality. ${ }^{17}$ It is a self-administered, non-disease specific scale for assessing information pertaining to not only sleep quality but also sleep quantity. The MOS-Sleep consists of 12 items related to sleep, and all questions are combined to form 6 subscales: Sleep Disturbance, Snoring, Short of Breath (awakening short of breath or with a headache), Sleep Adequacy, Daytime Somnolence, and Sleep Quantity. The Sleep Problems Index (SPI) is a composite score drawn from 9 items of four subscales, namely Sleep Disturbance, Short of Breath, Sleep Adequacy, and Daytime Somnolence. Responses to the individual questions were transformed to a $0-100$ scale according to manual instructions. ${ }^{18}$ With the exception of the Sleep Adequacy subscale, higher scores represent worse sleep outcomes. Sleep Quantity was based on the average number of hours of sleep per night during the previous 4 weeks. We used the Korean version of the MOSSleep Scale. ${ }^{17}$

\section{Quality of Life in Epilepsy 10}

The Quality of Life in Epilepsy (QOLIE)-10 consisted of 10 items and was derived from the QOLIE-31. ${ }^{19}$ The QOLIE-10 comprises seven components: five of them correspond to a single item from each of five subscales (seizure worry, overall QoL, emotional well-being, energy/fatigue, and cognitive functioning), one component includes two items on medication effects (physical effects and mental effects), and the last component includes three items on social function (work, driving, and social limits). Higher score in the QOLIE-10 indicates a better QoL in people with epilepsy. We used the Korean version of the QOLIE-10. ${ }^{20}$

\section{Hospital Anxiety and Depression Scale}

This scale consists of 14 items, 7 related to anxiety [Hospital Anxiety and Depression Scale-Anxiety (HADS-A) subscale] and 7 related to depression [HADS-Depression (HADS-D) sub- scale]. Each item has a 4-point response set, ranging from 0 for no distress to 3 for significant distress. ${ }^{21}$ For each subscale, the following cut-off points were used: $0-7$, normal; 8-10, mild disturbance; and 11-21, moderate to severe disturbance.

\section{Statistical Analyses}

To compare sleep problems between people with epilepsy and normal controls and to determine the relationships between clinical seizure variables and sleep problems, univariate analyses were performed using the student $t$ test, Pearson correlation analysis, and chi square test. To assess the impact of poor sleep quality measured by SPI on the QOLIE-10, linear regression analyses were performed in an unadjusted model (model 1), in model 2 (after controlling for demographic and seizure-related variables), and model 3 (after controlling additionally for depressive mood and anxiety). Variables that showed p-values $<0.05$ in the univariate linear regression analysis were included in a multiple linear regression analysis. Clinical variables tested were age, sex, and BMI. Seizure-related variables were age at seizure onset, epilepsy duration, epilepsy classification, dominant seizure type, frequency of both generalized and focal seizures, recurrence of generalized tonic-clonic seizure in the last two years, and AED treatments. Significance was set at $\mathrm{p}<0.05$. IBM Statistical Package for the Social Sciences 21.0 software (IBM Inc., Armonk, NY, USA) was used to perform these statistical analyses.

We also employed the mediational model, which proposes that poor sleep quality affect QoL via mediators such as depressive mood or anxiety. Evidence for mediation is considered sufficient if the relationship between sleep quality and QoL decreases when the effects of the mediators are controlled. Mediation effects were assessed using the Sobel test, which was performed using the R statistics program (version 2.14.2) and the "bda" package.

\section{RESULTS}

A total of 168 people with epilepsy were enrolled in this study. Their demographic and epilepsy-related characteristics are summarized in Table 1 and 2. The control group consisted of 56 employees at an university hospital. There were no differences in age and gender between the patient and control groups (Table 1). The patient group had a significantly higher BMI than the controls $(\mathrm{p}<0.001)$, and had a lower employment rate than the control group $(\mathrm{p}<0.001)$.

Among a total of 168 people with epilepsy, about 40\% ( $\mathrm{n}=$ 67) had been seizure-free for at least one year, whereas $60 \%(n=$ 101) had experienced seizures within the last year (Table 2). Sixteen percent $(n=27)$ reported having one or more seizures a month, and $15.5 \%(\mathrm{n}=26)$ had more than $90 \%$ of seizures during sleep. With regard to AED treatment, $47.6 \%(n=80)$ had been 
Table 1. Comparison of demographic features between epilepsy and control groups

\begin{tabular}{lccc}
\hline & Epilepsy $(\mathrm{n}=168)$ & Control $(\mathrm{n}=56)$ & $\mathrm{p}$ value \\
\hline Male/female, $\mathrm{n}$ & $77 / 91$ & $25 / 31$ & $\mathrm{NS}$ \\
Age, year, mean (SD) & $38.2(11.0)$ & $38.0(12.3)$ & $\mathrm{NS}$ \\
Body mass index, $\mathrm{kg} / \mathrm{m}^{2}(\mathrm{SD})$ & $26.8(6.8)$ & $22.5(3.5)$ & $<0.001$ \\
Employed, $\mathrm{n}(\%)$ & $118(72.0)$ & $56(100)$ & $<0.001$ \\
\hline
\end{tabular}

NS: not significant, SD: standard deviation.

Table 2. Characteristics of epilepsy in the participants $(n=168)$

\begin{tabular}{|c|c|}
\hline Seizure onset, years, mean (SD) & $22.7(13.6)$ \\
\hline Duration, years, mean (SD) & $15.7(9.3)$ \\
\hline \multicolumn{2}{|l|}{ Epilepsy syndrome, n (\%) } \\
\hline Idiopathic generalized & $29(17.3)$ \\
\hline Symptomatic partial & $45(26.8)$ \\
\hline Cryptogenic partial & $74(44.0)$ \\
\hline Undetermined & $20(6.5)$ \\
\hline \multicolumn{2}{|l|}{ Predominant seizure type, $\mathrm{n}(\%)$} \\
\hline Simple partial & $16(10.1)$ \\
\hline Complex partial & $75(47.5)$ \\
\hline Generalized tonic-clonic & $67(42.4)$ \\
\hline \multicolumn{2}{|l|}{ Seizure frequency, n (\%) } \\
\hline Remission for at least 1 year & $67(39.9)$ \\
\hline $1-11 /$ year & $71(44.0)$ \\
\hline$\geq 1 /$ month & $27(16.1)$ \\
\hline Nocturnal seizures (> 90\%) & $26(15.5)$ \\
\hline Monotherapy, n (\%) & $80(47.6)$ \\
\hline \multicolumn{2}{|l|}{ HADS-D subscale, n (\%) } \\
\hline$\leq 7$ & $91(58.0)$ \\
\hline $8-10$ & $37(23.6)$ \\
\hline$\geq 11$ & $29(18.5)$ \\
\hline \multicolumn{2}{|l|}{ HADS-A subscale, n (\%) } \\
\hline$\leq 7$ & $102(64.6)$ \\
\hline $8-10$ & $33(20.9)$ \\
\hline$\geq 11$ & $23(13.7)$ \\
\hline \multicolumn{2}{|l|}{ Antiepileptic drugs* } \\
\hline Valproic acid & $61(36.3)$ \\
\hline Lamotrigine & $53(31.5)$ \\
\hline Carbamazepine & $51(30.4)$ \\
\hline Oxcarbazepine & $43(25.6)$ \\
\hline Topiramate & $26(15.5)$ \\
\hline Levetiracetam & $23(13.7)$ \\
\hline Phenytoin & $19(11.3)$ \\
\hline
\end{tabular}

*Antiepileptic drugs prescribed for more than $10 \%$ of people with epilepsy.

SD: standard deviation, HADS-D: Hospital Anxiety Depression Scale-Depression subscale, HADS-A: Hospital Anxiety Depression Scale-Anxiety subscale. on monotherapy, and $52.4 \%(\mathrm{n}=88)$ had been on polytherapy. Valproic acid, lamotrigine, and carbamazepine were the most common drugs among the patients. Depressive mood and anxiety were observed in $42.0 \%(n=66)$ and $35.4 \%(n=56)$ of the patient group, respectively (Table 2).

\section{Comparison of Sleep Problems between Patient and Control Groups}

The prevalence of sleep problems are shown in Table 3. There were significant differences in insomnia symptoms: difficulty maintaining sleep (16.1\%) and 'wake up too early in the morning and cannot get back to sleep' responses (13.1\%) were more common in the patient group than in the control group $(5.4 \%$ and $1.8 \%$, respectively) (both $\mathrm{p}<0.05$ ). Difficulty initiating sleep was also more common in the patient group, but did not reach a statistically significant level $(p=0.093)$. There were no differences in the prevalence of EDS, OSA, and RLS between the patient and control groups (Table 3). In the MOS-Sleep Scale, patients had more sleep problems in Sleep Disturbance, Shortness of Breath, and SPI than the control group (all $\mathrm{p}<0.05$ ).

\section{Seizure-Related Factors Associated with Sleep Quality}

Age at seizure onset was negatively related to scores of SPI and Sleep Disturbance (both $\mathrm{p}<0.05$ ) (Table 4). Patients with simple partial seizures had higher SPI and Daytime Somnolence scores than those with complex partial or generalized tonic-clonic seizures (both $\mathrm{p}<0.05$ ). Patients without generalized tonic-clonic seizures for the last two years had more daytime sleepiness compared to those with generalized tonic-clonic seizures $(p<0.05)$. Absence of nocturnal seizures were significantly related to higher score of Daytime Somnolence than the presence of nocturnal seizures $(\mathrm{p}<0.05)$. Patients free of seizures for at least one year had more sleep quantity than those without seizure remission $(\mathrm{p}<0.05)$.

\section{The Effect of Poor Sleep Quality on QoL in People with Epilepsy}

Among the demographic and clinical seizure variables, age, seizure frequency, dominant seizure type, and AED treatment were significantly associated with QOLIE-10 scores. Unadjusted Model 1 showed a significant effect of SPI on lower QOLIE-10 scores ( $\mathrm{p}<0.001)$ (Table 5). After controlling for demographic and seizure variables (Model 2), SPI remained statistically sig- 
nificant and was identified as the only predictor for QOLIE-10. After controlling additionally for HADS-D and HADS-A (Model 3), however, SPI lost its statistical significance $(\beta=-0.021, \mathrm{p}=$ 0.769 ) and HADS-A, HADS-D, and polypharmacy were identified as the significant predictors for QOLIE-10 scores. SPI was related to HADS-D $(\mathrm{r}=0.447, \mathrm{p}<0.001)$ and HADS-A $(\mathrm{r}=$ $0.530, \mathrm{p}<0.001)$. The mediational model was tested to investigate HADS-D and HADS-A scores as mediators of the relation between SPI and QOLIE-10 scores. The Sobel test confirmed that the effect of SPI on QOLIE-10 was significantly mediated by

Table 3. Comparison of sleep disturbances between people with epilepsy and controls

\begin{tabular}{|c|c|c|c|}
\hline & Epilepsy $(n=168)$ & Control $(n=56)$ & $\mathrm{p}$ value \\
\hline \multicolumn{4}{|l|}{ Insomnia symptoms, $\mathrm{n}(\%)$} \\
\hline Difficulty initiating sleep & $31(18.5)$ & $5(8.9)$ & 0.093 \\
\hline Difficulty maintaining sleep & $27(16.1)$ & $3(5.4)$ & 0.041 \\
\hline Waking too early & $22(13.1)$ & $1(1.8)$ & 0.016 \\
\hline Excessive daytime sleepiness, $\mathrm{n}(\%)$ & $39(25.2)$ & $10(18.2)$ & 0.293 \\
\hline Epworth Sleepiness Scale, mean (SD) & $6.8(4.6)$ & $6.4(3.7)$ & 0.576 \\
\hline Obstructive sleep apnea, $\mathrm{n}(\%)$ & $32(21.5)$ & $8(14.3)$ & 0.247 \\
\hline Restless legs syndrome, $\mathrm{n}(\%)$ & $11(6.6)$ & $2(3.6)$ & 0.400 \\
\hline \multicolumn{4}{|c|}{ Medical Outcomes Study-Sleep Scale, mean (SD) } \\
\hline Sleep Disturbance & $26.0(24.3)$ & $18.6(15.3)$ & 0.009 \\
\hline Snoring & $25.9(33.1)$ & $23.2(24.9)$ & 0.573 \\
\hline Sleep Adequacy & $49.2(28.1)$ & $53.8(24.9)$ & 0.278 \\
\hline Shortness of Breath & $8.8(17.2)$ & $2.1(9.9)$ & $<0.001$ \\
\hline Daytime Somnolence & $29.3(20.5)$ & $24.4(17.1)$ & 0.110 \\
\hline Sleep Quantity, hr & $7.12(1.58)$ & $7.29(0.53)$ & 0.595 \\
\hline Sleep Problem Index & $28.9(18.0)$ & $23.6(12.0)$ & 0.014 \\
\hline
\end{tabular}

SD: standard deviation.

Table 4. Clinical seizure variables contributing to sleep disturbances

\begin{tabular}{|c|c|c|c|c|}
\hline MOS-Sleep Subscales & Clinical seizure variables & Mean (SD) & $\mathrm{r}$ & $\mathrm{p}$ value \\
\hline Sleep Disturbance & Age at seizure onset, yr & & -0.210 & 0.007 \\
\hline \multirow[t]{10}{*}{ Daytime Somnolence } & Nocturnal seizures & & & 0.040 \\
\hline & Yes & $21.6(13.7)$ & & \\
\hline & No & $30.8(21.4)$ & & \\
\hline & Seizure types & & & 0.013 \\
\hline & Simple partial & $44.2(27.2)$ & & \\
\hline & Complex partial & $28.9(19.6)$ & & \\
\hline & GTC & $27.6(19.1)$ & & \\
\hline & GTC seizures for 2 years & & & 0.025 \\
\hline & Absence & $34.7(23.0)$ & & \\
\hline & Presence & $27.0(18.8)$ & & \\
\hline \multirow[t]{3}{*}{ Sleep Quantity, h } & Seizure freedom for 1 year & & & 0.036 \\
\hline & No & $7.42(1.63)$ & & \\
\hline & Yes & $6.85(1.47)$ & & \\
\hline \multirow[t]{5}{*}{ Sleep Problem Index } & Seizure types & & & 0.025 \\
\hline & Simple partial & $39.0(17.9)$ & & \\
\hline & Complex partial & $27.5(17.6)$ & & \\
\hline & Generalized tonic-clonic & $25.8(16.7)$ & & \\
\hline & Age at seizure onset, yr & & -0.158 & 0.044 \\
\hline
\end{tabular}

SD: standard deviation, MOS-Sleep: Medical Outcomes Study-Sleep, GTC: generalized tonic-clonic. 
Table 5. Linear regression showing contributing factors to quality of life in people with epilepsy

\begin{tabular}{|c|c|c|c|c|}
\hline & \multicolumn{2}{|c|}{ Unstandardized coefficients } & \multirow{2}{*}{$\begin{array}{c}\text { Standardized coefficients } \\
\text { Beta }\end{array}$} & \multirow{2}{*}{$\mathrm{p}$ value } \\
\hline & B & SE & & \\
\hline \multicolumn{5}{|l|}{ Model 1* } \\
\hline SPI & -0.394 & 0.068 & -0.419 & $<0.001$ \\
\hline \multicolumn{5}{|l|}{ Model $2^{\dagger}$} \\
\hline SPI & -0.338 & 0.071 & -0.356 & $<0.001$ \\
\hline \multicolumn{5}{|l|}{ Model $3^{\ddagger}$} \\
\hline Polytherapy & -5.590 & 2.442 & -0.167 & 0.024 \\
\hline Anxiety & -1.861 & 0.349 & -0.467 & $<0.001$ \\
\hline Depression & -0.800 & 0.396 & -0.174 & 0.046 \\
\hline SPI & -0.021 & 0.073 & -0.023 & 0.769 \\
\hline
\end{tabular}

Dependent variable $=$ Quality of Life in Epilepsy-10.

${ }^{*}$ Unadjusted model. ${ }^{\dagger}$ Controlling for age, seizure frequency, dominant seizure type, and antiepileptic treatment. ${ }^{\ddagger}$ Controlling for age, seizure frequency, dominant seizure type, and antiepileptic treatment, depression, and anxiety.

SPI: Sleep Problem Index, SE: standard error.

both HADS-D $(\beta=-0.195, \mathrm{p}<0.001)$ and HADS-A $(\beta=-0.265$, $\mathrm{p}<0.001)$.

\section{DISCUSSION}

We performed a questionnaire-based study to evaluate the prevalence of sleep disturbances and their impact on QoL in people with epilepsy. Sleep disturbances, especially insomnia, were more common in the patient group than in the control group. Although sleep disturbance measured by SPI had no direct effects on QOLIE-10, it had an indirect effect on QOLIE-10 through HADS-D and HADS-A.

One of the clearly reproducible findings from previous studies $^{2-4}$ is that people with epilepsy, regardless of the syndrome, have many sleep-related complaints. In the present study, insomnia symptoms, especially difficulty maintaining sleep and waking up too early, were also more common in the patient group compared to controls. These findings are partially in agreement with the previous study ${ }^{5}$ showing that sleep maintenance insomnia was more frequent in epilepsy patients (52\%) than in controls (38\%) ( $\mathrm{p}=0.060)$. In addition, sleep disturbance, quantified by SPI of MOS-Sleep Scale, was found to be more severe in epilepsy patients than controls. In contrast to previous studies ${ }^{22}$ as well as to our studies, Khatami et al. ${ }^{5}$ showed that with few exceptions (sleep maintenance insomnia, nightmares, and bruxism), sleep-wake habits and frequency of sleep disorders were similar between people with epilepsy and controls. This result was probably because their control group, recruited from hospital staffs, may be at least somewhat biased by higher sleep complaints. In general, employees in Korea tend to sleep less than 7 hours a night, probably because they work very hard. This social trend of sleep insufficiency is not unusual among full-time employees in Korea. This trend was also found to be significant in the patient subgroup with and without seizure remission. Patients free of seizures for at least one year had shorter sleep times $(6.9 \mathrm{~h})$ than those who were not $(7.4 \mathrm{~h})$. Therefore, we excluded subjects with sleep time less than 7 hours a night from the control group.

We found an EDS prevalence of $25.2 \%$ in the patient group, and found no differences between the patient and control groups. In a recent literature review, ${ }^{23}$ the prevalence of EDS in epilepsy was found to vary from 10 to $48 \%$. In general, this prevalence was higher than $11.6 \%$ in the general population. ${ }^{24}$ Some studies ${ }^{25}$ found significant differences in the prevalence of EDS between the patient and control groups, but other studies ${ }^{5}$ did not. This discrepancy may be attributed to differences in patient samples.

Sleep Apnea of Sleep Disorder Questionnaire screening cutoffs that are slightly lower ( 29 for men and 26 for women) than those used in the general population may be more suitable for OSA diagnosis in epilepsy patients. ${ }^{15}$ Using these lower cutoffs, OSA prevalence in our sample was found to be $21.5 \%$, which was lower than the $28.2 \%$ to $35 \%$ in previous studies ${ }^{4,5,26}$ using the same cutoff scores in unselected epilepsy patients. The relatively lower BMI in our sample compared to western countries may also contribute to lower OSA prevalence in this study. In addition, it should be mentioned that the SA-SDQ is not a diagnostic tool and should only be used to screen individuals for OSA. ${ }^{13}$ Polysomnographic investigation of unselected adults with epilepsy found an OSA prevalence of $10.2 \%{ }^{27}$

Some seizure-related factors such as seizure types, recurrence of generalized tonic-clonic seizures within the last two years, seizure freedom for at least one year, and presence of nocturnal seizures were significantly related to one or more subscales of MOS-Sleep, but the direction of effect was the opposite of what we expected. For example, patients with simple partial seizures had higher SPI and Daytime Somnolence scores than 
those with complex partial or generalized tonic-clonic seizures. Patients without generalized tonic-clonic seizures within the last two years had more daytime sleepiness compared to those with generalized tonic-clonic seizures. There are few studies on the relationship between seizure-related factors and sleep disturbance. Xu et al. ${ }^{22}$ stated that patients who had experienced a seizure within the past week reported higher sleep problems than those with a less-recent seizure.

Sleep disturbances are known to have negative influences on mood and QoL in the general population. ${ }^{28,29}$ Epilepsy patients could be more susceptible to sleep disturbances because epilepsy itself is frequently associated with mood disturbances and impairment of QoL. ${ }^{30}$ We found a significant relationship between sleep disturbances and QoL, which was in agreement with previous studies. ${ }^{3,4}$ De Weerd et al. ${ }^{3}$ found that in partial epilepsy populations, patients with subjective sleep disturbances had lower mental and physical SF-36 QoL than those without sleep disturbances. The authors stated that these differences exceeded the thresholds for minimally important changes in epilepsy patients. Thus, they suggested that sleep disturbances in people with epilepsy adversely affect their QoL beyond that attributable to epilepsy and its treatment alone. Piperidou et al. ${ }^{4}$ also found that epilepsy patients with sleep disturbances, mainly those with insomnia, had significantly poorer QoL than those without sleep disturbances.

It is not well known whether sleep disturbances affect QoL independent of mood disturbance in people with epilepsy. In the present study, the effect of sleep disturbances, measured by the MOS Sleep Scale, on QoL lost its statistical significance after controlling for depressive mood and anxiety. This means that sleep disturbances did not have a direct effect on QoL in our sample. However, sleep disturbances were found to have indirect effects on QoL, mediated by depressive mood and anxiety symptoms. These findings were inconsistent with the recent study by Kwan et al., ${ }^{6}$ which employed a structural equation model to show that anxiety, depression, sleep disturbance, lack of seizure for four weeks prior to evaluation, and a number of current AEDs have direct (unmediated) effects on QoL. They also did not find an indirect effect of sleep disturbance on QoL through depressive symptoms, whereas symptoms of anxiety and sleep disturbance were mutually dependent. Their findings did not exclude the possibility of a relationship between depressive symptoms and sleep disturbance because interrelationships can change depending on the factors used to construct a structural equation model. Therefore, further studies are needed to determine how changes in one factor will affect others in a prospective longitudinal study.

Although sleep disturbances have been known to be more common in people with epilepsy than in healthy controls. ${ }^{2-4}$ it is unclear which and by how much clinical seizure variables affect sleep quality. We found no strong relationship between clinical seizure variables and sleep disturbances, quantified by the MOS
Sleep Scale. These findings were in agreement with the report by Xu et al. ${ }^{22}$ showing that the frequency of seizures within the past year or the number of AEDs did not have a statistically significant relationship with sleep disturbance. Exceptionally, they found that patients with a seizure within the past week had more sleep disturbances, quantified by the MOS Sleep Scale, than those whose most recent seizure occurred more than a week ago. ${ }^{22}$ One explanation for this finding may be attributed to the fact that the main limitation of the MOS Sleep Scale is its retrospective nature and the associated risk of recall biases. ${ }^{31}$ It may be that people with epilepsy recall and generalize their experiences beginning from the night that was disturbed by seizure recurrence in the past week, resulting in an overestimation of sleep disturbance.

This study has several limitations. First, formal and standardized diagnostic criteria were not used for sleep disturbance in this study. Second, the study population was derived from a group of patients receiving treatment at university hospitals. They likely have more severe seizures compared to people with epilepsy in primary clinics. Therefore, some findings of this study may not be generalizable to well controlled patients on monotherapy or patients with idiopathic generalized epilepsy. Third, AEDs are known to influence sleep structure and it is often suggested that sleep problems in people with epilepsy are at least partly due to AEDs. ${ }^{32}$ However, this study did not consider the effects of individual AEDs on sleep disturbances. Last, this study was based on a self-reported questionnaire. Therefore there is a risk of recall bias.

In conclusion, sleep disturbances, especially insomnia, are more common in people with epilepsy. Although sleep disturbance seems to have no direct effects on QoL, it has an indirect effect on QoL through depressive mood and anxiety symptoms in people with epilepsy. Therefore, more comprehensive efforts to avoid sleep deprivation and achieve good sleep quality are needed to improve QoL in people with epilepsy.

\section{Conflicts of Interest}

The authors have no financial conflicts of interest.

\section{REFERENCES}

1. Derry CP, Duncan S. Sleep and epilepsy. Epilepsy Behav 2013;26:394404.

2. Malow BA, Bowes RJ, Lin X. Predictors of sleepiness in epilepsy patients. Sleep 1997;20:1105-10.

3. de Weerd A, de Haas S, Otte A, Trenité DK, van Erp G, Cohen A, et al. Subjective sleep disturbance in patients with partial epilepsy: a questionnaire-based study on prevalence and impact on quality of life. Epilepsia 2004;45:1397-404.

4. Piperidou C, Karlovasitou A, Triantafyllou N, Terzoudi A, Constantinidis T, Vadikolias K, et al. Influence of sleep disturbance on quality of life of patients with epilepsy. Seizure 2008;17:588-94.

5. Khatami R, Zutter D, Siegel A, Mathis J, Donati F, Bassetti CL. Sleepwake habits and disorders in a series of 100 adult epilepsy patients--a prospective study. Seizure 2006;15:299-306.

6. Kwan P, Yu E, Leung H, Leon T, Mychaskiw MA. Association of sub- 
jective anxiety, depression, and sleep disturbance with quality-of-life ratings in adults with epilepsy. Epilepsia 2009;50:1059-66.

7. Alanis-Guevara I, Peña E, Corona T, López-Ayala T, López-Meza E, López-Gómez M. Sleep disturbances, socioeconomic status, and seizure control as main predictors of quality of life in epilepsy. Epilepsy Behav 2005;7:481-5.

8. Boylan LS, Flint LA, Labovitz DL, Jackson SC, Starner K, Devinsky O. Depression but not seizure frequency predicts quality of life in treatment-resistant epilepsy. Neurology 2004;62:258-61.

9. Johnson EK, Jones JE, Seidenberg M, Hermann BP. The relative impact of anxiety, depression, and clinical seizure features on health-related quality of life in epilepsy. Epilepsia 2004;45:544-50.

10. Lee SA, Lee SM, No YJ. Factors contributing to depression in patients with epilepsy. Epilepsia 2010;51:1305-8.

11. American Academy of Sleep Medicine. The international classification of sleep disorders: diagnostic \& coding manual. 2nd ed. Westchester, IL: American Academy of Sleep Medicine 2005.

12. Cho YW, Lee JH, Son HK, Lee SH, Shin C, Johns MW. The reliability and validity of the Korean version of the Epworth sleepiness scale. Sleep Breath 2011;15:377-84.

13. Douglass AB, Bornstein R, Nino-Murcia G, Keenan S, Miles L, Zarcone VP Jr, et al. The Sleep Disorders Questionnaire. I: Creation and multivariate structure of SDQ. Sleep 1994;17:160-7.

14. Kim B, Lee EM, Chung YS, Kim WS, Lee SA. The utility of three screening questionnaires for obstructive sleep apnea in a sleep clinic setting. Yonsei Med J 2015;56:684-90.

15. Weatherwax KJ, Lin X, Marzec ML, Malow BA. Obstructive sleep apnea in epilepsy patients: the Sleep Apnea scale of the Sleep Disorders Questionnaire (SA-SDQ) is a useful screening instrument for obstructive sleep apnea in a disease-specific population. Sleep Med 2003;4:51721.

16. Walters AS. Toward a better definition of the restless legs syndrome. The International Restless Legs Syndrome Study Group. Mov Disord 1995;10:634-42.

17. Kim MK, You JA, Lee JH, Lee SA. The reliability and validity of the Korean version of the Medical Outcomes Study-Sleep Scale in patients with obstructive sleep apnea. Sleep Med Res 2011;2:89-95.

18. Spritzer KL, Hays RD. MOS Sleep Scale: a manual for use and scoring, version 1.0. Los Angeles, CA: RAND, 2003.
19. Cramer JA, Perrine K, Devinsky O, Meador K. A brief questionnaire to screen for quality of life in epilepsy: the QOLIE-10. Epilepsia 1996; 37:577-82.

20. Lee SA, Yun SC, Lee BI; Korean QoL in Epilepsy Study Group. Reliability and Validity of the Korean Version of QOLIE-10 in Epilepsy. J Clin Neurol 2006;2:238-45.

21. Zigmond AS, Snaith RP. The hospital anxiety and depression scale. Acta Psychiatr Scand 1983;67:361-70.

22. Xu X, Brandenburg NA, McDermott AM, Bazil CW. Sleep disturbances reported by refractory partial-onset epilepsy patients receiving polytherapy. Epilepsia 2006;47:1176-83.

23. Giorelli AS, Passos P, Carnaval T, Gomes Mda M. Excessive daytime sleepiness and epilepsy: a systematic review. Epilepsy Res Treat 2013; 2013:629469.

24. Soldatos CR, Allaert FA, Ohta T, Dikeos DG. How do individuals sleep around the world? Results from a single-day survey in ten countries. Sleep Med 2005;6:5-13.

25. Sanchez de Leon I, Foldvary N, Marsilio D, Dinner DS. Effect of seizure frequency and antiepileptic drugs on daytime alertness as measured by the Epworth Sleepiness Scale in patients with epilepsy. Epilepsia 1997;38:56.

26. Malow BA, Levy K, Maturen K, Bowes R. Obstructive sleep apnea is common in medically refractory epilepsy patients. Neurology 2000;55: 1002-7.

27. Manni R, Terzaghi M, Arbasino C, Sartori I, Galimberti CA, Tartara A. Obstructive sleep apnea in a clinical series of adult epilepsy patients: frequency and features of the comorbidity. Epilepsia 2003;44:836-40.

28. Harris M, Glozier N, Ratnavadivel R, Grunstein RR. Obstructive sleep apnea and depression. Sleep Med Rev 2009;13:437-44.

29. Yang EH, Hla KM, McHorney CA, Havighurst T, Badr MS, Weber S. Sleep apnea and quality of life. Sleep 2000;23:535-41.

30. Sandstrom SA, Bowman ES, Johnson CS, Salanova V. Interictal mood disorder and quality of life in active epilepsy. Epilepsy Behav 2010;17: 199-204.

31. Kopec JA, Esdaile JM. Bias in case-control studies. A review. J Epidemiol Community Health 1990;44:179-86.

32. Manni R, Terzaghi M. Comorbidity between epilepsy and sleep disorders. Epilepsy Res 2010;90:171-7. 